\title{
INTERIOR AND POLYNOMIAL EXTENSIONS OF IMMERSED CIRCLES
}

\author{
MORRIS L. MARX ${ }^{1}$ AND ROGER F. VERHEY 2
}

1. Introduction. A mapping $f$ from one topological space to another is said to be open if the image of every open set is open; it is light if the preimage of every point is totally disconnected; it is interior if it is light and open. Let $R^{2}$ denote the oriented plane, let $S^{1}$ denote the unit circle in $R^{2}$, let $S^{2}$ denote the oriented two-sphere with a distinguished point " $\infty$," and let $E^{2}$ denote the closed unit disk in $R^{2}$. An interior extension of a mapping $\delta: S^{1} \rightarrow R^{2}$ is a mapping $f: E^{2} \rightarrow S^{2}$ such that $f$ is sense-preserving and interior on the open two-cell and $f \mid S^{1}=\delta$. If $f$ is an interior extension of $\delta: S^{1} \rightarrow R^{2}$ and $f(a)=\infty$, then $a$ is called a pole of $f$.

A mapping $\delta: S^{1} \rightarrow R^{2}$ is called a regular immersion if $\delta$ is $C^{1}$ and $\delta^{\prime}(t) \neq 0$ on $S^{1}$. An image point $\delta(t)$ is called a vertex if $\delta(t)$ has exactly two preimages and the corresponding tangent vectors are linearly independent. A regular immersion is called a normal immersion (Whitney [12, p. 281]) if it has a finite number of vertices and all other image points have exactly one pre-image. The tangent winding number $\tau(\delta)$ of a normal immersion $\delta$ is defined to be the topological degree of the Gauss map $\delta^{\prime}(t) /\left|\delta^{\prime}(t)\right|$ of $S^{1}$ into $S^{1}$. Let $n(\delta)$ denote the number of vertices of $\delta$.

Titus [8] has given an algorithm which determines when a normal immersion $\delta$ has an interior extension $f: E^{2} \rightarrow R^{2}$. For those normal immersions which have interior extensions without poles, Marx [5] determines the minimum number of branch points needed and their multiplicities. This is part of the problem (Morse [6, p. 76]) of determining the branch points, poles, and zeros of interior mappings of a two-cell into a two-sphere. In this paper we show that every normal immersion $\delta: S^{1} \rightarrow R^{2}$ has an interior extension $f: E^{2} \rightarrow S^{2}$ with at most one pole and such that the multiplicity of this pole is at most $\frac{1}{2}(n(\delta)-\tau(\delta)+1)$. Thus, by the Stoilow-Whyburn Theorem [7, p. $121]$ or $\left[13\right.$, p. 103], given any normal immersion $\delta, S^{1}$ can be reparametrized in such a way that $\delta$ has a meromorphic extension with one pole of multiplicity at most $\frac{1}{2}(n(\delta)-\tau(\delta)+1)$. This improves a

Received by the editors November 22, 1968 and, in revised form, May 15, 1969.

${ }^{1}$ Research supported by the University Research Council of Vanderbilt University.

${ }^{2}$ Research supported by a University of Michigan Faculty Research Fellowship. A portion of the contents of this paper is based on the author's doctoral dissertation written under the direction of Professor C. Titus of the University of Michigan. 
result due to Morse [6, p. 74] which shows that to every regular analytic closed curve $\Gamma$ there exists a meromorphic function $G$ on $E^{2}$ such that the image of $S^{1}$ under $G$ is $\Gamma$. His proof, however, gives no control on the number of zeros, poles, or branch points of $G$. We further prove that if a normal immersion $\delta$ admits an interior extension with no poles, then $S^{1}$ can be reparametrized in such a way that $\delta$ has a polynomial extension of degree less than or equal to $\frac{1}{2}(n(\delta)+\tau(\delta)+1)$.

Notation and definitions will be as in [4] unless otherwise specified.

\section{Main results.}

Definition 1. Suppose $\delta: S^{1} \rightarrow R^{2}$. Let $p$ be a point on the outer boundary of $\delta$. Suppose $C$ is a Jordan curve in $R^{2}$ such that [ $\delta$ ] $C$ Ins $C$ and pick $q \in C$. Let $\gamma$ be a parametrization of an arc from $p$ to $q$ such that $[\gamma] \cap([\delta] \cup C)=\{p, q\}$. Suppose $h$ is a homeomorphism of $S^{1}$ onto $C$. Let $h^{n}$ denote the curve $\sum_{i=1}^{n} q q(h)$; that is, $h^{n}$ is the $n$-fold product of $h$ with itself with the usual product for paths. Define the curve $\eta=\delta$ aug $n, h, \gamma, p, q$ by

$$
\eta=h^{n}+q p(-\gamma)+p p(\delta)+p q(\gamma) .
$$

We will suppress any of $h, \gamma, p, q$ in the notation if it is clear from the context what they are. We define $\delta$ aug $0, h, \gamma, p, q$ to be the mapping $\delta$.

Definition 2. If $\delta: S^{1} \rightarrow R^{2}$ has an interior extension to $E^{2}$ that maps into $R^{2}$, i.e., the extension has no poles, then $\delta$ is an interior boundary.

Theorem 1. Let $\delta: S^{1} \rightarrow R^{2}$ be normal and suppose $h, \gamma, p$, $q$ are as in Definition 1 . Then there exists a nonnegative integer $m \leqq \frac{1}{2}(n(\delta)-\tau(\delta)+1)$ such that $\delta$ aug $m, h, \gamma, p, q$ is an interior boundary.

Proof. The proof is by induction on $n(\delta)$, the number of vertices of $\delta$.

If $n(\delta)=0$, then $\delta$ describes either a positively or negatively oriented Jordan curve. Suppose the former. Then $\delta=\delta$ aug 0 is an interior boundary and the conclusion follows. Now suppose $\delta$ describes a negatively oriented Jordan curve. Let

$$
D=\left\{z \in R^{2}|1 \leqq| z \mid \leqq 2, \operatorname{Im} z \geqq 0\right\}
$$

and let $f: D \rightarrow R^{2}$ be defined by $f(z)=z^{2}$. Then $f \mid$ Boundary $D$ describes a map topologically equivalent to $\delta$ aug $1, h, \gamma, 1,4$ and

$$
1 \leqq \frac{1}{2}(n(\delta)-\tau(\delta)+1)=\frac{1}{2}(0-(-1)+1) .
$$

Suppose the theorem is true for any normal mapping with $<n$ vertices. Let $\delta: S^{1} \rightarrow R^{2}$ be normal with $n$ vertices. 
Select a Jordan curve $C$ with $[\delta] C$ Ins $C$ and let $h$ be a homeomorphism of $S^{1}$ onto $C$. Pick $p$ on the outer boundary of $\delta$ and let $q \in C$. Suppose $\gamma$ is a parametrization of an arc from $p$ to $q$. Beginning at $p$, trace along $\delta$ until the first vertex, say $r$, is encountered. Then $r$ will also be on the outer boundary of $\delta$. Let $r_{1}$ and $r_{2}$ be the two preimages of $r$ under $\delta$ on $S^{1}$. These two points divide $S^{1}$ into two arcs $A_{1}$ and $A_{2}$; say that $A_{1}$ is the arc that contains the point $f^{-1}(p)$. Let $g_{j}$ be a mapping of $A_{j}$ onto $S^{1}$ that is one-to-one on $A_{j}-\left\{r_{1}, r_{2}\right\}$ with $g_{j}\left(r_{1}\right)=g_{j}\left(r_{2}\right), j=1,2$. Then $\delta^{j}=\delta \circ g_{j}^{-1}$ is a normal mapping with $n_{j}<n$ vertices, $j=1,2$. (Actually, $\delta^{j}$ is not differentiable at $g_{j}\left(r_{1}\right)$, but we smooth the corner to obtain a normal mapping.) We note here that $n_{1}+n_{2} \leqq n-1$ and $\tau\left(\delta^{1}\right)+\tau\left(\delta^{2}\right)=\tau(\delta)$.

Let $\beta$ be a parametrization of the arc $r p(-\delta)$. By the induction hypothesis, there exist nonnegative integers $m_{1}$ and $m_{2}$ such that $m_{j} \leqq \frac{1}{2}\left(n_{j}-\tau\left(\delta^{j}\right)+1\right), j=1,2$, and $\delta^{1}$ aug $m_{1}, h, \gamma, p, q$ and $\delta^{2}$ aug $m_{2}$, $h, \beta^{+} \gamma, r, q$ are interior boundaries. Then there exist disks $D_{1}$ and $D_{2}$ and interior mappings $f_{j}: D_{j} \rightarrow R^{2}, j=1,2$, such that $f_{1}$ extends $\delta^{1}$ aug $m_{1}$ and $f_{2}$ extends $\delta^{2}$ aug $m_{2}$.

We must make cases depending on whether $m_{2}=0$ or $m_{2}>0$, i.e., whether $\delta^{2}$ is or is not an interior boundary. First assume $m_{2}>0$.

If $m_{1}>0$, take $K$ to be the arc of Boundary $D_{1}$ such that $f_{1} \mid K$ describes the curve $q p(-\gamma)+p r(\delta)$ and take $L$ to be the arc of Boundary $D_{2}$ such that $f_{2} \mid L$ describes the curve $r p(-\delta)+p q(\gamma)$. If $m_{1}=0$, take $K$ to be the arc of Boundary $D_{1}$ such that $f_{1} \mid K$ describes the curve $\operatorname{pr}(\delta)$ and take $L$ to be the arc of Boundary $D_{2}$ such that $f_{2} \mid L$ describes the curve $r p(-\delta)=r p(\beta)$. In either case, let $D$ be the disk obtained as a decomposition space of $D_{1} \cup D_{2}$, whose nondegenerate elements are all sets of the form $\{x, y\}$, where $x \in K, y \in L$, and $f_{1}(x)=f_{2}(y)$. Let $\phi: D_{1} \cup D_{2} \rightarrow D$ be the natural projection. Define a map $f: D \rightarrow R^{2}$ by $f(z)=f_{j} \phi^{-1}(z)$, where $j=1$ if $z \in \phi\left(D_{1}\right)$ and $j=2$ if $z \in \phi\left(D_{2}\right)$. Then $f$ is interior [10, Theorem 9, p. 336] and extends $\delta$ aug $m_{1}+m_{2}, h, \gamma, p, q$.

Now we consider the case where $\delta^{2}$ is an interior boundary, i.e., where $m_{2}=0$. The method of gluing $D_{1}$ and $D_{2}$ together used in the previous paragraph will not work here and a different method is needed. First we note that $\delta^{2}$ can only be an interior boundary when

$$
\left|\begin{array}{ll}
\zeta^{\prime}(b) & \eta^{\prime}(b) \\
\zeta^{\prime}(a) & \eta^{\prime}(a)
\end{array}\right|>0
$$

where $\delta(t)=\zeta(t)+i \eta(t), a$ is the first preimage of $r$ encountered as the circle is traversed in the positive orientation from $\delta^{-1}(p)$, and $b$ is the second preimage of $r$ so encountered. Intuitively speaking, the above 
condition means that standing at $p$ facing along $\delta$ in the direction that $\delta$ is going, the curve crosses transversely at $r$ from left to right. If the above determinant is negative, then points near $r$ have negative winding number with respect to $\delta^{2}$; hence $\delta^{2}$ cannot be an interior boundary, as interior boundaries have nonnegative winding number. Let $A$ be an arc of $S^{1}$ containing $a$ such that $\delta$ maps $A$ homeomorphically; similarly chose $B$ containing $b$. Let $\epsilon:[0,1] \rightarrow R^{2}$ be such that $[\epsilon] \cap[\delta]=\{r\}$ and such that $[\epsilon]$ lies to the left of $\delta(A)$ and to the left of $\delta(B)$ (see Figure 1). By choosing $\epsilon$ this way, it will lie to the left of both $\delta^{1}$ and $\delta^{2}$ at $r$.

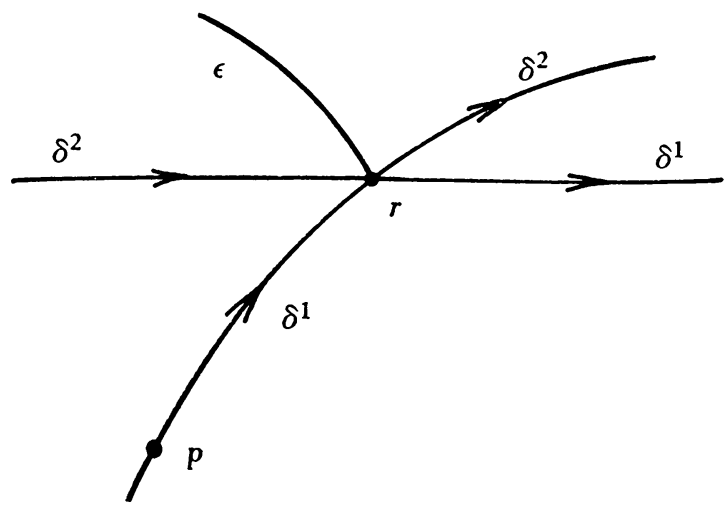

Figure 1

By the induction hypothesis, $\delta^{1}$ aug $m_{1}$ is an interior boundary. Also, we are assuming that $\delta^{2}$ is an interior boundary. Without loss of generality we may suppose that there exist interior mappings $f_{j}: E^{2} \rightarrow R^{2}, j=1,2$, such that $f_{1}$ extends $\delta^{1}$ aug $m_{1}$ and $f_{2}$ extends $\delta^{2}$. Since $\epsilon$ lies to the left of both $\delta^{1}$ and $\delta^{2}$ at $r$, we may use [4, Theorem 1 , p. 49] to claim the existence of $\operatorname{arcs} T_{1}$ and $T_{2}$ in $E^{2}$ such that, for $j=1$ or $2, T_{j}$ intersects $S^{1}$ at one end point and $f_{j}$ maps $T_{j}$ homeomorphically onto $[\epsilon]$. Again without loss of generality assume that $T_{1}$ $=T_{2}=\left\{z \in E^{2} \mid z\right.$ real, $\left.0 \leqq z \leqq 1\right\}$. Furthermore, we assume that for $z$ in $T_{1}=T_{2}, f_{1}(z)=f_{2}(z)$. Then we define a map $f$ on $E^{2}$ as follows:

$$
\begin{aligned}
f(z) & =f_{1}\left(z^{2}\right) & & \text { if } \operatorname{Im} z \geqq 0, \\
& =f_{2}\left(z^{2}\right) & & \text { if } \operatorname{Im} z \leqq 0 .
\end{aligned}
$$

This map is well defined and hence, continuous. It is open, except possibly on the real axis; thus, it is open on the interior of $E^{2}$ by [10, 
Theorem 9, p. 336]. Finally, $f$ extends a curve topologically equivalent to $\delta$ aug $m_{1}, h, \gamma, p, q$.

To complete the proof of the theorem, we only have to show that $m_{1}+m_{2} \leqq \frac{1}{2}(n(\delta)-\tau(\delta)+1)$. As noted before, $n_{1}+n_{2} \leqq n(\delta)-1$ and $\tau\left(\delta^{1}\right)+\tau\left(\delta^{2}\right)=\tau(\delta)$. Thus,

$$
\begin{aligned}
m_{1}+m_{2} & \leqq \frac{1}{2}\left(n_{1}-\tau\left(\delta^{1}\right)+1\right)+\frac{1}{2}\left(n_{2}-\tau\left(\delta^{2}\right)+1\right) \\
& \leqq \frac{1}{2}\left[\left(n_{1}+n_{2}\right)-\left(\tau\left(\delta^{1}\right)+\tau\left(\delta^{2}\right)\right)+2\right] \\
& \leqq \frac{1}{2}[n(\delta)-1-\tau(\delta)+2] \\
& \leqq \frac{1}{2}(n(\delta)-\tau(\delta)+1) .
\end{aligned}
$$

Definition. Let $A$ be a closed annulus in $R^{2}$ bounded by Jordan curves $C_{1}$ and $C_{2}$ with $C_{1} \subset$ Ins $C_{2}$. Suppose $\delta^{1}$ and $\delta^{2}$ are mappings of $S^{1}$ into $R^{2}$. We say that $\left(\delta^{2}, \delta^{1}\right)$ is an a-boundary if there exist homeomorphisms $H_{j}: S^{1} \rightarrow C_{j}, j=1,2$, and a mapping $f: A \rightarrow R^{2}$ such that $f$ is interior on the interior of $A$, and $f \circ H_{j}=\delta^{i}, j=1,2$.

Corollary 1. Suppose $\delta: S^{1} \rightarrow R^{2}$ is regular. Then there exist a homeomorphism $H: E^{2} \rightarrow E^{2}$ and a mapping $g: E^{2} \rightarrow S^{2}$ such that $g$ is meromorphic on the open two-cell, $g$ has exactly one pole, and $\delta=$ $(g \circ H) \mid S^{1}$. Furthermore, if $\delta$ is normal, then $g$ can be taken to have this pole of order $m$, where $m \leqq \frac{1}{2}(n(\delta)-\tau(\delta)+1)$.

Proof. By [2, Proposition 1, p. 264], there exists a normal mapping $\delta^{*}$ such that $\left(\delta, \delta^{*}\right)$ is an $a$-boundary. From the theorem it follows that $\delta^{*}$ aug $\mathrm{m}, h$ is an interior boundary, where $m \leqq \frac{1}{2}\left(n\left(\delta^{*}\right)-\tau\left(\delta^{*}\right)+1\right)$. Thus, $\left(h^{m},-\delta^{*}\right)$ is an $a$-boundary [4, Lemma 5.1, p. 50] and by Lemma 5.3 of that same paper, $\left(\delta^{*},-h^{m}\right)$ is an $a$-boundary. It is an easy consequence of $\left[10\right.$, Theorem 9, p. 336] that $\left(\delta,-h^{m}\right)$ is an $a$-boundary. Let $A, H_{1}, H_{2}, f$ be as in the definition of $a$-boundary for the pair $\left(\delta,-h^{m}\right)$. There exists a mapping $p$, continuous on $C_{1}$ $\cup$ Ins $C_{1}$, interior on Ins $C_{1}$, such that $p$ is topologically equivalent to the meromorphic mapping $w=z^{-m}$ and $p$ agrees with $-h^{m} \circ H_{1}^{-1}$ on $C_{1}$. Then we define the interior mapping $\phi$ from $D=C_{2} \cup$ Ins $C_{2}$ into $S^{2}$ by $\phi=p$ on Ins $C_{1}, \phi=f$ otherwise. Note that $\phi^{-1}(\infty)$ is a branch point of multiplicity $m-1$. Since $\delta$ is locally $1-1$, we can take $\phi$ to be locally $1-1$ in a neighborhood of Boundary $D$. It follows from the Remarks of $\left[1\right.$, p. 88], that there is a homeomorphism $G_{1}: D \rightarrow R^{2}$, and a function $\psi$, continuous on $G_{1}(D)$, meromorphic on the interior of $G_{1}(D)$, such that $\phi=\psi \circ G$. By the Riemann Mapping Theorem and a theorem of Caratheodory (see $[8$, p. 46]), there exists an analytic homeomorphism $G_{2}: G_{1}(D) \rightarrow E^{2}$. The homeomorphism $H_{1}$ extends to a homeomorphism of $E^{2}$ onto $D$. On $S^{1}$, we have $\delta=f \circ H_{1}$ 
$=\phi \circ H_{1}=\psi \circ G_{1} \circ H_{1}=\psi \circ G_{2}^{-1} \circ G_{2} \circ G_{1} \circ H_{1}$. Take $g=\psi \circ G_{2}^{-1}$ and $H=G_{2} \circ G_{1} \circ H_{1}$; note that $g$ has a pole of order $m$. In case that $\delta$ is normal, we take $\delta^{*}=\delta$ and $m \leqq 1 / 2(n(\delta)-\tau(\delta)+1)$.

REMARK 1. Corollary 1 generalizes Theorem 20.4 of [6].

REMARK 2. If $\delta: S^{1} \rightarrow R^{2}$ is normal, then Corollary 1 guarantees the existence of a mapping $f: E^{2} \rightarrow S^{2}$ that is interior on the open two cell and extends $\delta$. We can ask the following: Given any such extension of $\delta$, what is the minimal possible number of branch points and poles? It is probably true that an extension with exactly one pole does not have the minimal number of branch points and poles. The first author has solved this problem in the case that $f$ has no poles (see [5]).

REMARK 3. For each interior extension $f$ of $\delta$, let $N_{f}(\infty)$ be the sum of the multiplicities of the poles of $f$. Let $n_{\delta}(\infty)$ denote the minimum of $N_{f}(\infty)$ taken over all interior extensions $f$ of $\delta$. Thus, $n(\delta) \geqq 2 n_{f}(\infty)$ $+\tau(\delta)-1$. In [11] the second author considers numerical invariants for diffeomorphically equivalent normal immersions. Two normal immersions $\delta$ and $\eta$ are said to be diffeomorphically equivalent if there exist sense-preserving diffeomorphisms $h: S^{1} \rightarrow S^{1}$ and $H: S^{2} \rightarrow S^{2}$ such that $\delta=H \circ \eta \circ h$. Let $E$ be an equivalence class of diffeomorphically equivalent normal immersions and let $p=\operatorname{Min}_{f \in E} n_{f}(\infty)$. Since $\tau$ and $n$ are numerical invariants [11], the theorem shows that $n \geqq 2 p$ $+\tau-1$. Thus, the inequality is independent of the parametrization chosen.

REMARK 4. For each natural number $n$ there exists a normal immersion $\delta$ for which the inequality of Corollary 1 is exact $\left(N_{f}(\infty)\right.$ can be arbitrarily large). This can be seen by considering the normal immersions represented in Figure 2 for the cases $n=1,2$, and 3 . The generalization to any $n$ is obvious.

COROLlaRY 2. Let $\mu$ be the number counting multiplicities of branch point antecedents of an interior mapping $f$ of the open two-cell into $S^{2}$. If $\delta$ is a normal immersion of $S^{1}$ into $R^{2}$, then there exists an interior extension $f$ of $\delta$ such that $\mu \leqq n(\delta)$.

Proof. By a theorem of Morse [6, p. 71], $2 N_{f}(\infty)-\mu=1-\tau(\delta)$. Substituting the inequality of Corollary 1 into this equation, we obtain the desired result.

Corollary 3. If $\delta: S^{1} \rightarrow R^{2}$ is a normal interior boundary, then there exists a complex polynomial $P$ of degree $m \leqq \frac{1}{2}(n(\delta)+\tau(\delta)+1)$ and a homeomorphism $H: R^{2} \rightarrow R^{2}$ such that $\delta=(P \circ H) \mid S^{1}$.

Proof. By Theorem $1,-\delta$ aug $m, h$ is an interior boundary, where 


$$
\begin{aligned}
m & \leqq \frac{1}{2}(n(-\delta)-\tau(-\delta)+1) \\
& =\frac{1}{2}(n(\delta)+\tau(\delta)+1) .
\end{aligned}
$$

Thus, it follows from $\left[4\right.$, Lemma 5.1, p. 50] that $\left(h^{m}, \delta\right)$ is an $a$-boundary. Suppose then that $A=\{z|1 \leqq| z \mid \leqq 2\}$ and $f: A \rightarrow R^{2}$ is a light open extension of $\left(h^{m}, \delta\right)$ to $A$. We can find a map $\psi$ of $S^{2}-\{z|| z \mid \leqq 2\}$ into $S^{2}$ that is topologically equivalent to $z^{m}$ and that agrees with $h^{m}$ on $|z|=2$. Let $g$ be an extension of $\delta$ to $E^{2}$. Then define $\phi: S^{2} \rightarrow S^{2}$ by $\phi=g$ on $E^{2}, \phi=f$ on $A$, and $\phi=\psi$ elsewhere; $\phi$ is an interior mapping. It follows from [1] that there is a homeomorphism $H: S^{2} \rightarrow S^{2}$ and an analytic function $P: S^{2} \rightarrow S^{2}$ such that $P \circ H=\phi$. We may assume without loss of generality that $H(\infty)=\infty$ and so $P(\infty)=\infty$. From this we see that $P$ is a polynomial [3, Theorem 8.5, p. 217]; since $P$ has a branch point of multiplicity $m-1$ at $\infty, P$ must have degree $m$.

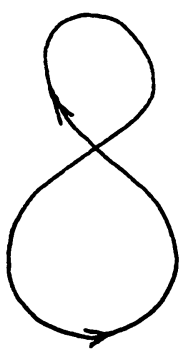

$n=1$

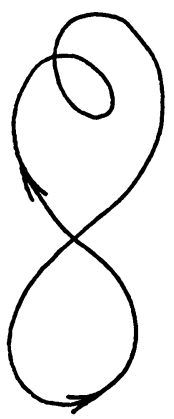

$n=2$

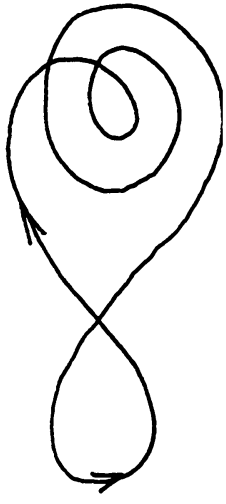

$n=3$

FIGURE 2

REMARK. Titus $[8$, p. 61] asks a related and perhaps more difficult question. Characterize all normal $\delta: S^{1} \rightarrow R^{2}$ with the property that there exists a homeomorphism $H: S^{1} \rightarrow S^{1}$ and polynomial $P$ of degree $m$ such that $\delta=P \circ H$. Titus conjectures that such a $\delta$ has at most $(m-1)^{2}$ vertices.

3. A folded ribbon theorem. In this section we use Theorem 1 to obtain an analogue of the Folded Ribbon Theorem of G. Francis [2].

Definition. Suppose $\alpha, \beta: S^{1} \rightarrow R^{2}$ are differentiable mappings. A differentiable increasing homotopy from $\alpha$ to $\beta$ is a differentiable mapping $H: S^{1} \times I \rightarrow R^{2}$ such that 
(1) $H(x, 0)=\alpha(x)$ all $x \in S^{1} ; H(x, 1)=\beta(x)$ all $x \in S^{1}$.

(2) $H$ is locally one-to-one on a neighborhood of $S^{1} \times\{0,1\}$.

(3) The Jacobian of $H$ is nonnegative.

In case the Jacobian of $H$ is strictly positive, we call $H$ a positive monotone regular homotopy (= monotopy).

The definition of a differentiable increasing homotopy is equivalent to the one given in [9]; this equivalence can be established using the results of that paper.

THEOREM [9]. There exists a differentiable increasing homotopy from $\alpha$ to $\beta$ if and only if $(\alpha, \beta)$ is an a-boundary.

Francis [2] proves the following theorem, called the Folded Ribbon Theorem.

Theorem. Given two regular mappings $\alpha, \beta: S^{1} \rightarrow R^{2}$ with the same tangential winding number, there exist a regular mapping $\eta: S^{1} \rightarrow R^{2}$ and monotopies $G$ and $H$ such that $G$ is a monotopy from $\alpha$ to $\eta$ and $H$ is a monotopy from $\beta$ to $\eta$.

We prove here an analogue of the Folded Ribbon Theorem replacing monotopy by differentiable increasing homotopy.

Theorem 2. Given two regular mappings $\alpha, \beta: S^{1} \rightarrow R^{2}$, there exist a differentiable mapping $\eta: S^{1} \rightarrow R^{2}$, a differentiable increasing homotopy $G$ from $\alpha$ to $\eta$, and a differentiable increasing homotopy $H$ from $\beta$ to $\eta$.

Proof. By the above mentioned theorem of Titus we need only find a mapping $\eta$ such that $(\alpha, \eta)$ and $(\beta, \eta)$ are $a$-boundaries. It follows from [2, Proposition 1, p. 264] that there exist normal mappings $\alpha^{*}$ and $\beta^{*}$ such that $\left(\alpha, \alpha^{*}\right)$ and $\left(\beta, \beta^{*}\right)$ are $a$-boundaries. By Theorem 1 and Lemma $5.3\left[4\right.$, p. 54] $\left(\alpha^{*},-h_{1}^{n}\right)$ and $\left(\beta^{*},-h_{2}^{m}\right)$ are $a$-boundaries for $h_{1}$ and $h_{2}$ describing suitably chosen Jordan curves and $n$ and $m$ positive integers. Say that $m \geqq n$. Without loss of generality we may assume that the image of $h_{1}$ is contained in the bounded component of the plane complement of the image of $h_{2}$. It is a straightforward induction argument to verify that $\left(h_{2}^{m}, h_{1}^{n}\right)$ is an $a$-boundary; whence, $\left(-h_{1}^{n},-h_{2}^{m}\right)$ is an $a$-boundary. Using [10, Theorem 9, p. 336], if $\left(\alpha, \alpha^{*}\right),\left(\alpha^{*},-h_{1}^{n}\right),\left(-h_{1}^{n},-h_{2}^{m}\right)$ are $a$-boundaries, then $\left(\alpha,-h_{2}^{m}\right)$ is an $a$-boundary. Similarly $\left(\beta,-h_{2}^{m}\right)$ is an $a$-boundary. Take $\eta=-h_{2}^{m}$ and the theorem is proved.

\section{REFERENCES}

1. P. T. Church, Extensions of Stoïlow's theorem, J. London Math. Soc. 37 (1962), 86-89. MR $26 \# 337$. 
2. G. K. Francis, The folded ribbon theorem for regular closed curves in the plane, Bull. Amer. Math. Soc. 74 (1968), 264-267. MR 36 \#3329.

3. E. Hille, Analytic function theory. Vol. 1: Introduction to higher mathematics, Ginn, Boston, Mass., 1959. MR 21 \#6415.

4. M. L. Marx, Normal curves arising from light open mappings of the annulus, Trans. Amer. Math. Soc. 120 (1965), 45-56. MR 33 \#3278.

5. - The branch point structure of extensions of interior boundaries, Trans. Amer. Math. Soc. 131 (1968), 79-98. MR 36 \#5914.

6. M. Morse, Topological methods in the theory of functions of a complex variable, Ann. of Math. Studies, no. 15, Princeton Univ. Press, Princeton, N. J., 1947. MR 9, 20.

7. S. Stoillow, Leçons sur les principes topologiques de la thêorie des fonctions analytiques, 2nd ed., Gauthier-Villars, Paris, 1956. MR 18, 568.

8. C. J. Titus, The combinatorial topology of analytic functions on the boundary of a disk, Acta Math. 106 (1961), 45-64. MR 29 \#3652.

9. - Characterizations of the restriction of a holomorphic function to the boundary of a disk, J. Analyse Math. 18 (1967), 351-359. MR 35 \#3072.

10. C. J. Titus and G. S. Young, The extension of interiority, with some applications, Trans. Amer. Math. Soc. 103 (1962), 329-340. MR 25 \#559.

11. R. F. Verhey, Diffeomorphic invariants of immersed circles (to appear).

12. H. Whitney, On regular closed curves in the plane, Compositio Math. 4 (1937), 276-284.

13. G. T. Whyburn, Topological analysis, 2nd rev. ed., Princeton Math. Series, no. 23. Princeton Univ. Press, Princeton, N. J., 1964. MR 29 \#2758.

VANDERBILT UNIVERSITY AND

University of Michigan, Dearborn Campus 